\title{
Eating a Balanced Diet: A Healthy Life through a Balanced Diet in the Age of Longevity
}

\author{
Soo Lim* \\ Department of Internal Medicine, Seoul National University Bundang Hospital, Seoul National University College of Medicine, Seongnam, Korea
}

Recently, trends in the Korean diet have favored westernized and unbalanced meals that mainly provide a single nutrient. To stop this unfavorable trend, the Committee of the Ten Guidelines for a Health Life: Korean Medical Association released three best practices to follow for the healthy and balanced diet in 2017. The purpose of these guidelines is to encourage people to eat a balanced diet that meets the recommended caloric intake, thereby reducing the risk of obesity and obesity-related diseases. First, eat a carbohydrate:protein:fat ratio of 55:20:25. Middle-aged and elderly Koreans consume a high proportion of carbohydrates as part of their total caloric intake, and those $\geq 65$ years consume a low proportion of fat as part of the total caloric intake. Second, reduce consumption of soda and sugar-sweetened beverages. The recent World Health Organization recommendation for added sugar is less than $10 \%$ of a person's total daily energy intake ( $<50 \mathrm{~g} /$ day). Koreans currently consume $72.1 \mathrm{~g}$ of added sugar per day. Koreans between the ages of 6 and 29 years consume sugar mostly through sodas, and those $\geq 30$ years consume sugar mostly through coffee. Third, maintain the recommended caloric intake for a healthy diet. Rapid weight gain increases blood sugar levels and blood pressure, which can lead to diabetes and hypertension, as well as increasing the risk of cardiovascular disease, stroke, and colorectal cancer. To help prevent these conditions, people should maintain a healthy weight by avoiding overeating and being physically active starting at a young age.

Key words: Beverage, Obesity, Metabolic syndrome, Body weight

\author{
${ }^{*}$ Corresponding author \\ Soo Lim \\ iD \\ https://orcid.org/0000-0002-4137-1671 \\ Department of Internal Medicine, Seoul \\ National University Bundang Hospital, \\ Seoul National University College of \\ Medicine, 82 Gumi-ro 173beon-gil, \\ Bundang-gu, Seongnam 13620, Korea \\ Tel: +82-31-787-7035 \\ Fax: +82-31-787-4051 \\ E-mail:limsoo@snu.ac.kr
}

\section{INTRODUCTION}

The Korean Medical Association (KMA) is South Korea's largest and most-trusted decision-making organization, made up of Korean physicians. The 35th KMA Congress in 2017 set its focus as "Future Medicine and Healthy Aging." With the goal of establishing a new medical paradigm that advocates for the public interests and fosters social awareness. To achieve this goal, a plan was made to publish "Ten Guidelines for a Healthy Life: Korean Medical Association statement (2017)." These guidelines are: (1) quitting smoking, (2) drinking in moderation, (3) eating a balanced diet, (4) being physically active, (5) having a regular sleep schedule,
(6) thinking positively, (7) receiving routine health screenings and immunizations, (8) managing stress, (9) paying attention to particulate matter and emerging infectious disease, and (10) avoiding excessive exposure to mobile devices.

This manuscript has been pressed as the chapter 3: Eating a Balanced Diet from Ten Guidelines for a Healthy Life published by KMA in 2017. It has been modified for the format of Journal of Obesity \& Metabolic Syndrome. The TFT Committee of the Ten Guidelines for a Healthy Life: Korean Medical Association statement (2017) and Korean Society for the Study of Obesity approved this publication to advocate public awareness and education. 


\section{EAT A CARBOHYDRATE:PROTEIN:FAT RATIO OF 55:20:25}

\section{Consume meals that satisfy the ideal carbohydrate, protein, and fat ratio}

A balanced diet following the recommended energy and nutrient intake range is desirable for chronic disease prevention and management. According to the Dietary Reference Intakes values for Koreans (a report on recommended energy and nutrient intake for improving health and preventing disease in the Korean population) reported by the Ministry of Health and Welfare and the Korean Nutrition Society, a healthy diet should consist of $55 \%-65 \%$ carbohydrates, $7 \%-20 \%$ protein, and $15 \%-30 \%$ fat (for individuals 3 years old and older). ${ }^{1,2}$ Especially in Korea, the middle-aged and elderly over 50 years old have too high a rate of carbohydrate intake and those over 65 years old have too low a rate of fat intake. These dietary habits underscore the need for a balanced diet. ${ }^{1}$ Accordingly, this publication, Ten Guidelines for a Healthy Life: Korean Medical Association statement (2017), proposes a nutrient intake ratio in which carbohydrate intake would be reduced and protein intake would be increased.
The food balance wheels of the Korean Dietary Reference Intakes (Fig. 1) emphasize the importance of a balanced diet, sufficient fluid intake, and regular physical activity for maintaining health. In short, regularly following the recommended caloric intake, consuming a balanced distribution of nutrients, and engaging in an adequate amount of physical activity can prevent obesity and lifestyle-related diseases. ${ }^{2}$

\section{Avoid diet plans which are not supported by scientific} evidence, such as the low-carb high-fat diet

People have shown great interest in the low-carb high-fat (LCHF) diet, which was recently introduced and promoted in newspapers and media as having excellent weight loss results. However, this trend has caused controversy and led to a shortage of butter and increased consumer consumption of pork belly in Korea. The LCHF diet reduces carbohydrate intake to $5 \%-10 \%$ of the recommended total caloric intake, while increasing fat intake to $70 \%$ or more. This diet plan is too extreme and is an abnormal way to eat. ${ }^{3}$

The purported effectiveness of this extreme form of LCHF diet that spread throughout media outlets everywhere has not clearly been proven. Moreover, health problems can occur if a person fol-

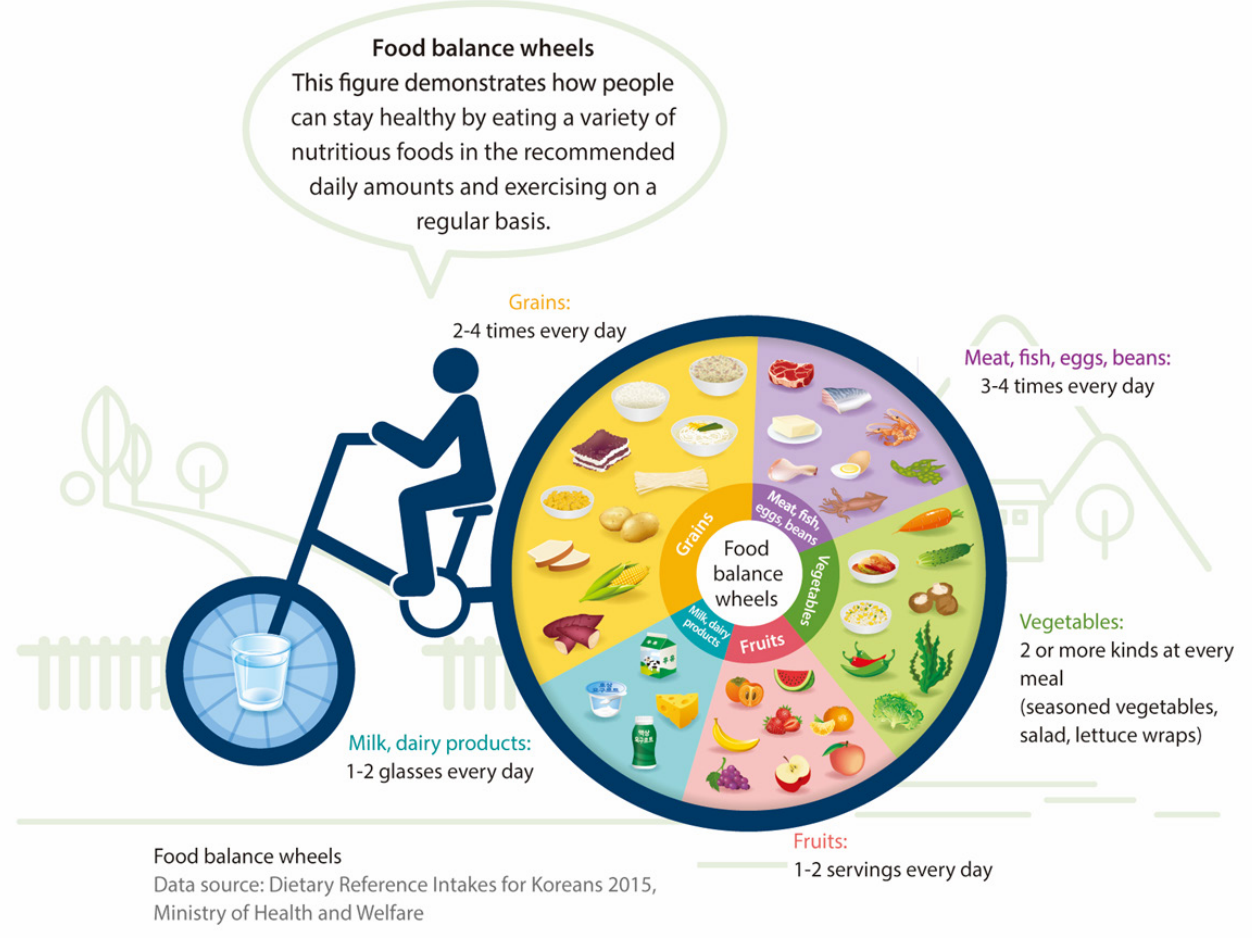

Figure 1. Food balance wheels. Adapted from Ministry of Health and Welfare and the Korean Nutrition Society. ${ }^{2}$ 
lows this diet for a prolonged period. For example, excessive intake of food products high in fat, especially saturated fat, can increase the levels of low-density lipoprotein (LDL)-cholesterol (bad cholesterol) in the blood, thereby increasing the risk of cardiovascular disease. When following an abnormally high-fat diet, it becomes difficult to consume an appropriate variety of foods, resulting in an imbalance of micronutrients and decreased fiber intake, which in turn changes the intestinal microbial flora in ways that cause oxidative stress and an increased inflammatory response. Extreme restriction of carbohydrates, meanwhile, increases ketone body synthesis, which negatively affects muscles and bones and reduces glucose levels, causing side effects such as decline in concentration. ${ }^{3}$

\section{Patients with diabetes, hypertension, or cardiovascular}

\section{disease need to discuss their diet with a physician}

Patients being treated for diabetes, hypertension, and cardiovascular disease need to pay extra attention to their dietary choices. Single-nutrient diets such as the LCHF diet can have harmful effects on disease management in patients with uncontrolled diabetes or with heart or kidney problems. Furthermore, if a patient taking diabetes medication suddenly reduces his or her carbohydrate intake, it may cause hypoglycemia, or even worse, ketosis. These diseases could also be exacerbated if the blood LDL cholesterol level increases due to a LCHF diet. Thus, the ground rule is to have these patients maintain a balanced diet as part of an individually customized diet therapy plan that has been discussed with their physicians. ${ }^{3}$

\section{Consume balanced meals with various foods}

Consuming a diet with balanced nutrient intake is not only necessary to prevent and manage chronic diseases, but also essential for children and adolescents to ensure their healthy development. It is crucial to eat an appropriate-neither excessive nor deficient-amount from each food group. For this reason, the Ministry of Health and Welfare recently announced dietary guidelines to help manage obesity, hypertension, and dyslipidemia in Korea. The guidelines suggest eating balanced meals, including a variety of foods such as rice, grains, vegetables, fruits, milk and other dairy products, meats, fish, eggs, and beans. ${ }^{1}$

According to the Korea National Health and Nutrition Examina- tion Survey, calcium intake, the main source of which is milk or dairy products, continues to be insufficient, with individuals consuming only two-thirds of the current 2015 recommended intake amount. More serious intake deficiencies were prevalent in people with lower socioeconomic status, suggesting that more attention should be paid to this demographic. In contrast, the amount of sodium intake was 2.2 times higher than the recommended daily allowance $(2,000 \mathrm{mg})$ in men and 1.6 times higher in women. It is estimated that four out of five people consume more sodium than recommended. An additional investigation of the intake of various food groups revealed that, until 2014, individuals' daily intake of grains gradually decreased, while daily meat consumption rose steadily. Vegetable and fruit intake, in comparison, was still inadequate, with only $38.3 \%$ of people eating more than $500 \mathrm{~g}$ of vegetables and fruits daily (based on data in 2014). This percentage was even lower in people with lower socioeconomic status, meaning that a systematic investigation is needed..$^{1,4}$

Regarding the excessive or insufficient intake of various nutrients and food groups in Korea, consumption practices on the individual level should be changed first; in addition, strategies for improving nutrition management are needed and can be developed using dietary policies that support the centralized management of nutrients and food groups. The Health Plan 2020 (2016-2020) set the nutrition-specific objective of increasing the percentage of the population with healthy dietary habits, including the consumption of appropriate amounts of dietary fat and sodium, fruits and vegetables, calcium, and vitamin A, and the outcomes of this strategy are eagerly anticipated. ${ }^{5}$

\section{REDUCE CONSUMPTION OF SODA AND SUGAR-SWEETENED BEVERAGES}

Recently, the World Health Organization recommended that added sugar should account for less than $10 \%$ of total daily energy intake, and that the intake level should be lower than $5 \%$ in some circumstances, depending on the conditions of member states. As the recommended daily caloric intake of an average adult is 2,000 $\mathrm{kcal}$, the daily amount of added sugar recommended by the World Health Organization is estimated to be $50 \mathrm{~g}$ or less. Based on these guidelines, Korea recommends that the total sugar intake should be 


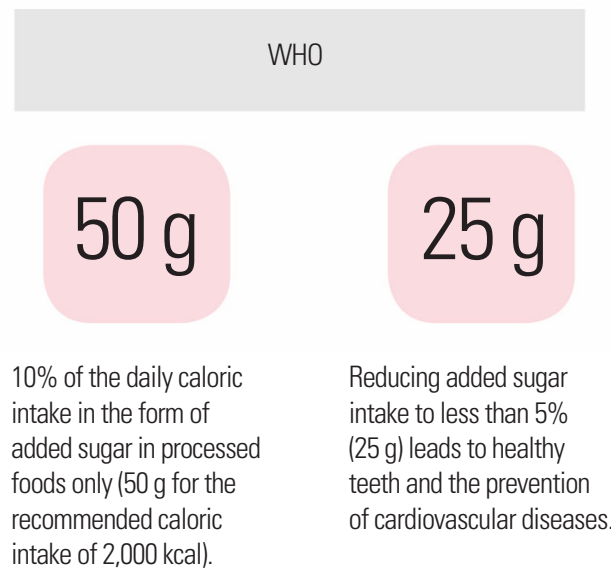

Dietary Reference

Intakes for Koreans

$100 \mathrm{~g}$

Total sugar intake, including both natural and added sugar, should be less than $20 \%$ of the daily caloric intake (100 g for the recommended caloric intake of 2,000 kcal).

Figure 2. Daily intake recommendations for adults. ${ }^{2,6}$ WHO, World Health Organization.

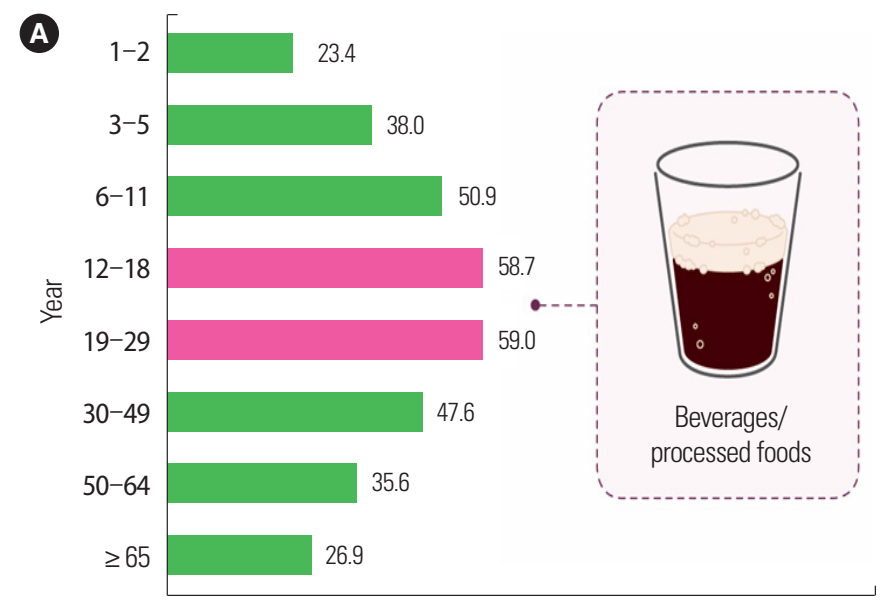

Sugar intake $(g)$

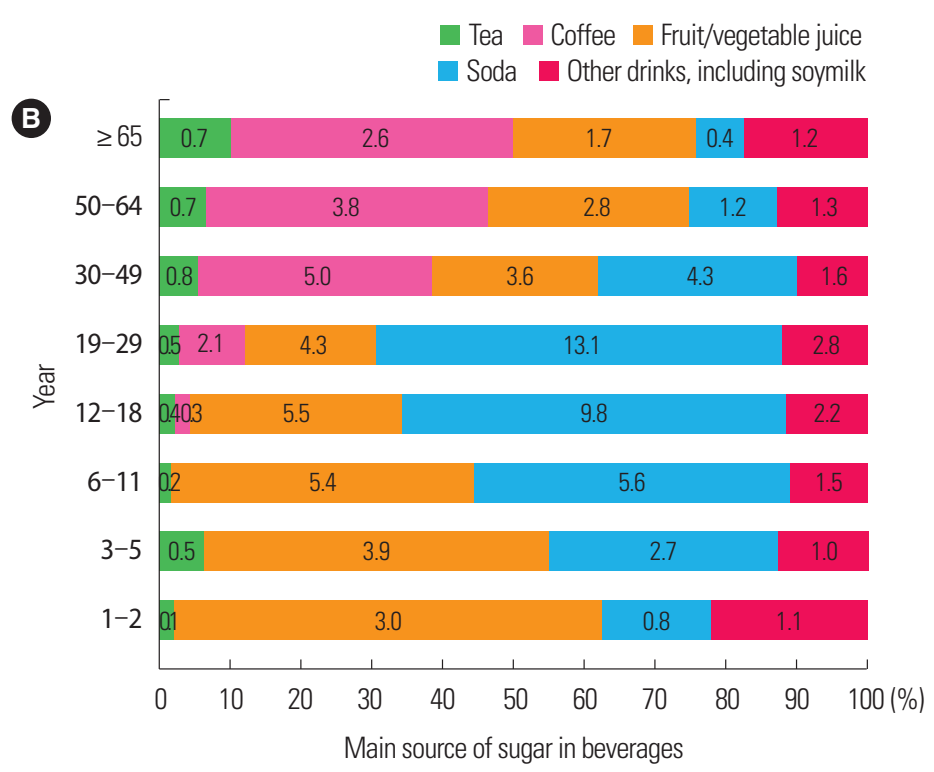

Figure 3. (A) Amount of sugar intake through processed foods by age group. (B) Distribution of main sources of sugar in beverages.?

$10 \%-20 \%$ of the total daily energy intake, and that added sugar should be less than $10 \%$ (Fig. 2)., ${ }^{2,6}$ Added sugars are monosaccharides, disaccharides, and syrups that are added to foods when they are processed or prepared. The main sources of added sugar are sugar, high-fructose corn syrup, starch syrup, molasses, honey, syrup, and fruit juice concentrate (Fig. 3). ${ }^{7}$

The Ministry of Food and Drug Safety reported that the total sugar intake for Koreans was $72.1 \mathrm{~g}$ (less than $20 \%$ of the recommended daily caloric intake for a 2,000 kcal diet), which is currently not a level of huge concern; however, there was a $3.5 \%$ yearly average increase between 2007 to 2013, and the amount of sugar in- take from processed foods is currently $44.7 \mathrm{~g}$ (2013), reflecting a yearly average increase of $5.8 \%$ compared to $2007 .{ }^{1}$ Accordingly, the Ministry of Food and Drug Safety has proposed sugar-related recommendations, such as "Eat less sugar" and "Drink plenty of water instead of sweetened drinks" (Fig. 4). ${ }^{1}$ Considering the increase in sugar intake, along with the rapid increase in beverage consumption, it is time to take preemptive and systematic actions to encourage appropriate levels of sugar intake by encouraging Koreans to change their dietary habits. Additionally, according to an investigation conducted by the Ministry of Food and Drug Safety, people between the ages of 3 and 29 years old exceeded the recom- 


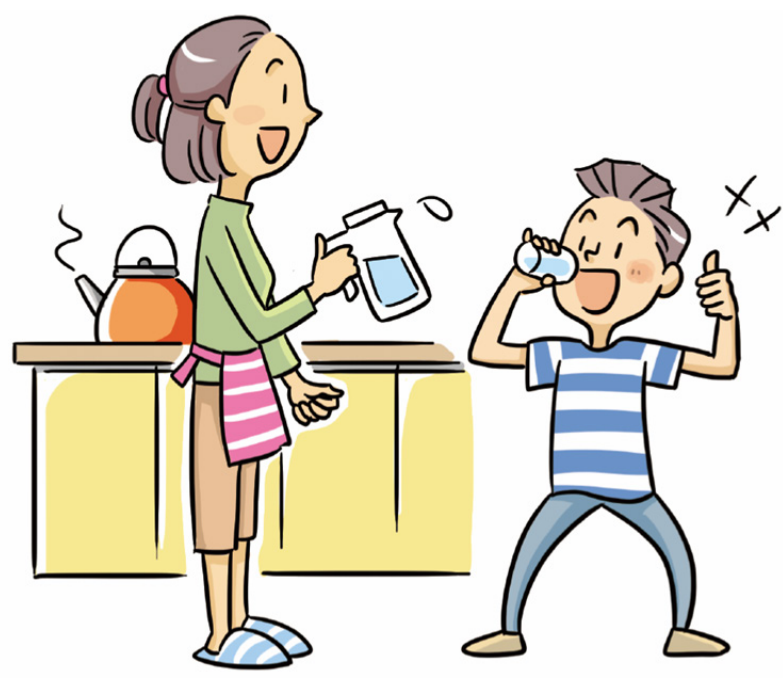

Amount of daily sugar intake through beverages: $8.7 \mathrm{~g}$ (2007) $\rightarrow 13.9 \mathrm{~g}$ (2013)

Figure 4. Drink plenty of water instead of sweetened drinks.

mended amount of sugar intake through processed foods in 2013, and the number of people from other age groups who exceeded this amount was on the rise. Presumably, added sugar intake through beverages or processed food was the cause for this increase. Those aged 6 years and older mostly consumed sugar from processed foods through beverages. The investigation reported that 1 - to 5-year-olds mostly consumed sugar through fruit and vegetable juices, 6- to 29-year-olds through soda, and those 30 years old and over through coffee, especially coffee with added sugar, such as coffee mixes and canned coffee. ${ }^{1}$

Sugar-sweetened beverages are quite diverse, including drinks such as soda, vitamin drinks, energy drinks, and coffee, and these drinks account for a large part of the daily sugar intake of teenagers. Teenagers have a particularly high sugar intake compared to other age groups; therefore, continuous monitoring and management of their sugar consumption are necessary. We need to provide consumer education to raise awareness of the significant amounts of added sugar present in children's food, such as juice, processed milk such as chocolate milk, yogurt, and snacks.

\section{MAINTAIN THE RECOMMENDED CALORIC INTAKE FOR A HEALTHY DIET}

\section{Keep watching your weight}

The prevalence of adult obesity in Korea in 2015 is two in five men and one in four women, as defined by a body mass index of $25 \mathrm{~kg} / \mathrm{m}^{2}$ or higher. For women, statistics show that the rate is higher in people with lower socioeconomic status. ${ }^{8}$ Obesity has a strong association with cardiovascular disease and stroke, which increases the risk of mortality, as well as with other causes of death, including cancer. Therefore, obesity should be regarded as an important public health and socioeconomic issue. In particular, abdominal fat accumulation is associated with glucose intolerance, and increased body fat reduces insulin sensitivity. Furthermore, the fact that hypertension is more common in centrally obese people than peripherally obese people indicates that the distribution of body fat is more important than simply measuring obesity. $\mathrm{Ab}$ dominal obesity is associated with higher serum triglyceride and LDL-cholesterol levels, lower high-density lipoprotein (HDL)cholesterol levels, and a higher frequency of accompanying hyperinsulinemia. ${ }^{9}$

The rapid increase in obesity rates in Korea was caused by the shift to westernized dietary habits and decreased physical activity due to advances in transportation methods. These causes led to an imbalance between energy intake and metabolism, which ultimately caused a rapid increase in obesity, cardiovascular disease, diabetes, and obesity-related cancer (for example, colorectal cancer). Therefore, to reduce the obesity rate, national education programs and government policies that encourage people to consistently maintain the recommended caloric intake and to increase their physical activity level are vital. ${ }^{10}$ According to the 2014 Korea Health Statistics, the energy intake rate steadily increased starting in 2004, while the walking rate gradually decreased, meaning that an action plan is needed. ${ }^{4}$ Additionally, being aware of one's own weight and examining one's own behaviors related to obesity can help fight obesity. ${ }^{11}$

\section{Maintain a healthy weight from childhood onward}

Obesity is the main factor that causes metabolic syndrome, which is a common health issue. When children and teenagers have metabolic syndrome, they are likely to develop diabetes, hyperlipidemia, and cardiovascular disease. Since these diseases require long-term treatment and management, they can lead to other health concerns and increased medical expenditures. Therefore, the increase of metabolic syndrome in teenagers could become a 
critical public health and socioeconomic issue affecting the future of Korea. In particular, among the five components of metabolic syndrome (high glucose, high blood pressure, high triglycerides, low HDL-cholesterol, and abdominal obesity), there was a decrease in HDL-cholesterol levels (10.5\%) and increases in triglyceride levels (6.2\%) and abdominal obesity (2.9\%), which indicates that the lack of physical activity and excessive nutrient intake can be considered the main causes of the increase in metabolic syndrome among children and teenagers. ${ }^{12}$ In fact, it is important to note that over the past 10 years, Korean teenagers have been engaging in less exercise and consuming more fat. ${ }^{1}$

A comparative analysis of National Health and Nutrition Examination Survey data from the United States and Korea in the past 10 years showed a decrease in the prevalence of metabolic syndrome among teenagers in the United States, while the rate doubled for Korean teenagers. ${ }^{12}$ If the prevalence of metabolic syndrome among Korean teenagers continues to rise, it is predicted that metabolic syndrome will increase by $0.4 \%$ every year, resulting in 22,000 new cases annually among teenagers. To prevent this, schools and households should provide balanced meals, increase the number of physical education classes, and provide after-school programs that allow teenagers to voluntarily engage in more physical activity, all of which require parents to change their perceptions of their children's health and for a different governmental policy approach to be implemented. ${ }^{12}$

\section{Slow down weight gain when overweight or obese}

According to a recent study, the larger and faster the weight gain compared to one's weight at the age of 20 , the higher the risk of developing cardiovascular disease (Fig. 5). Thus, attention should

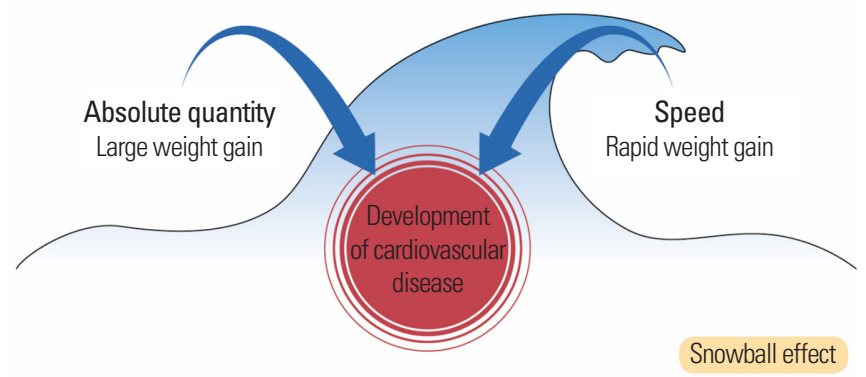

Figure 5. Conditions of weight gain and risks of cardiovascular disease: the snowball effect. also be paid to obesity early in life and to the rate and speed of weight gain. ${ }^{13}$ Rapid weight gain causes insulin resistance, increases inflammatory responses, and raises blood sugar and blood pressure, fundamentally damaging intravascular endothelial cells, which could cause coronary artery disease. In other words, the snowball effect of rapid and significant weight gain increases the risks of cardiovascular disease; therefore, it is important for people in their 20s and 30s to control their weight.

Currently, the prevalence of diabetes and cardiovascular disease is continuously increasing in Korea. This could lead to major issues on the individual and social levels. It is important to implement preventive strategies, such as reducing westernized, high-fat, and high-calorie dietary habits, increasing physical activity, and maintaining a healthy weight early in life. Unless behavioral changes are made regarding westernized dietary habits and decreased physical activity, medical costs will rise.

\section{CONCLUSION}

Recently, trends in the Korean diet have favored westernized and unbalanced meals. To stop this unfavorable trend, the Committee of the Ten Guidelines for a Health Life: Korean Medical Association released three best practices to follow for the healthy and balanced diet in 2017. (1) Eat a carbohydrate:protein:fat ratio of 55:20:25. (2) Reduce consumption of soda and sugar-sweetened beverages. (3) Maintain the recommended caloric intake for a healthy diet. The purpose of these guidelines is to encourage people to eat a balanced diet that meets the recommended caloric intake, thereby reducing the risk of obesity and obesity-related diseases.

\section{CONFLICTS OF INTEREST}

The author declares no conflict of interest.

\section{ACKNOWLEDGMENTS}

We thank Yu Jin Paek, Department of Family Medicine, Hallym University Sacred Heart Hospital; Mi-Kyung Sung, Department of Food and Nutrition, Sookmyung Women's University; Hye Ran 
Yang, Department of Pediatrics, Seoul National University Bundang Hospital; and Jung Eun Lee, Department of Food and Nutrition, Seoul National University.

\section{REFERENCES}

1. Ministry of Health and Welfare. Government establishes National Dietary Guidelines for Koreans [Internet]. Sejong: Ministry of Health and Welfare; 2016 [cited 2018 Feb 27]. Available from: http://www.mohw.go.kr/react/al/sal0301vw.jsp? PAR_MENU_ID $=04 \& M E N U \_I D=0403 \& C O N T$ SEQ $=$ 330959\&page $=1$

2. Ministry of Health and Welfare; The Korean Nutrition Society. Dietary Reference Intakes for Koreans 2015. Sejong: Ministry of Health and Welfare; 2015.

3. Korean Endocrine Society; Korean Diabetes Association; Korean Society for the Study of Obesity; The Korean Nutrition Society; The Korean Society of Lipid and Atherosclerosis. Joint statement on the low-carb high-fat diet trend from 5 professional organizations [Internet]. Seoul: Korean Society for the Study of Obesity; 2016 [cited 2018 Feb 27]. Available from: http://www.kosso.or.kr/board/view.html?num=1123\&start=0 $\&$ code $=$ notice_list \&comm $=\&$ key $=$ subject $\&$ keyword $=\% B 0 \% \mathrm{~F}$ $8 \%$ B5\%BF\&left=\&left comm=

4. Korea Centers for Disease Control and Prevention. Korea Health Statistics 2014: Korea National Health and Nutrition Examination Survey (KNHANES VI-2). Cheongju: Korea Centers for Disease Control and Prevention; 2015.

5. Ministry of Health and Welfare; Korea Health Promotion Foundation. Health plan 2020 (2016-2020). Seoul: Korea Health Promotion Foundation; 2015.

6. World Health Organization. Guideline: sugars intake for adults and children [Internet]. Geneva: World Health Organization;
2015 [cited 2018 Feb 27]. Available from: http://www.who. int/nutrition/publications/guidelines/sugars_intake/en/

7. Korea Health Industry Development Institute. Sugar database compilation for commonly consumed foods. Cheongju: Ministry of Food and Drug Safety; 2015.

8. Korea Centers for Disease Control and Prevention. Korea Health Statistics 2015: Korea National Health and Nutrition Examination Survey (KNHANES VI-3). Cheongju: Korea Centers for Disease Control and Prevention; 2016.

9. Kim SR, editor. Obesity and metabolic syndrome. Proceedings of the fall conference of Korean Society for Health Promotion and Disease Prevention; 2013 Nov 2; Seoul, Korea. Seoul: Korean Society for Health Promotion and Disease Prevention; 2013.

10. Lim S, Park KS, Lee HK, Cho SI; Korean National Health and Nutrition Examination Surveys. Changes in the characteristics of metabolic syndrome in Korea over the period 19982001 as determined by Korean National Health and Nutrition Examination Surveys. Diabetes Care 2005;28:1810-2.

11. Ministry of Health and Welfare. Becoming informed about obesity [Internet]. Sejong: Ministry of Health and Welfare; 2013 [cited 2018 Mar 5]. Available from: http://www.mohw. go.kr/react/al/sal0101vw.jsp?PAR_MENU_ID=04\&MENU_ $\mathrm{ID}=040101 \&$ CONT_SEQ $=292313 \&$ page $=1$

12. Lim S, Jang HC, Park KS, Cho SI, Lee MG, Joung H, et al. Changes in metabolic syndrome in American and Korean youth, 1997-2008. Pediatrics 2013;131:e214-22.

13. Lim S, Choi SH, Kim KM, Choi SI, Chun EJ, Kim MJ, et al. The association of rate of weight gain during early adulthood with the prevalence of subclinical coronary artery disease in recently diagnosed type 2 diabetes: the MAXWEL-CAD study. Diabetes Care 2014;37:2491-9. 